\title{
SPIN PHYSICS AT RHIC - A THEORETICAL OVERVIEW
}

\author{
MARCO STRATMANN \\ Inst. for Theor. Physics, Univ. of Regensburg, D-93040 Regensburg, Germany \\ E-mail: marco.stratmann@physik.uni-regensburg.de
}

\begin{abstract}
We review how RHIC is expected to deepen our understanding of the spin structure of longitudinally and transversely polarized nucleons. After briefly outlining the current status of spin-dependent parton densities and pointing out open questions, we focus on theoretical calculations and predictions relevant for the RHIC spin program. Estimates of the expected statistical accuracy for such measurements are presented, taking into account the acceptance of the RHIC detectors.
\end{abstract}

\section{Lessons from (Un)polarized DIS}

Before reviewing the prospects for spin physics at the BNL-RHIC we briefly turn to longitudinally polarized deep-inelastic scattering (DIS) and what we have learned from twenty years of beautiful data ${ }^{1}$. Figure 1 compares the available information on the DIS structure function $g_{1}\left(x, Q^{2}\right)$ to results of a typical next-to-leading order (NLO) QCD fit. From such types of analyses a pretty good knowledge of certain combinations of different quark flavors has emerged, and it became clear that quarks contribute only a small fraction to the proton's spin. However, there is still considerable lack of knowledge regarding the polarized gluon density $\Delta g$, which is basically unconstrained by present data, the separation of quark and antiquark densities and of different flavors, and the orbital angular momentum of quarks and gluons inside a nucleon. In addition, spin effects with transverse polarization at the leading-twist level, the so-called 'transversity' densities, have not been measured at all. With the exception of orbital angular momentum RHIC can address all of these questions as will be demonstrated in the following ${ }^{2}$.

There is also an important difficulty when analyzing polarized DIS data in terms of spin-dependent parton densities: compared to the unpolarized case the presently available kinematical coverage in $x$ and $Q^{2}$ and the statistical precision of polarized DIS data are much more limited ${ }^{1}$. As a consequence, one is forced to include data into the fits from $\left(x, Q^{2}\right)$-regions where fits of unpolarized leading-twist parton densities start to break down, see Fig. 1. Data from RHIC, taken at 'resolution' scales $Q^{2}$ where perturbative QCD and 


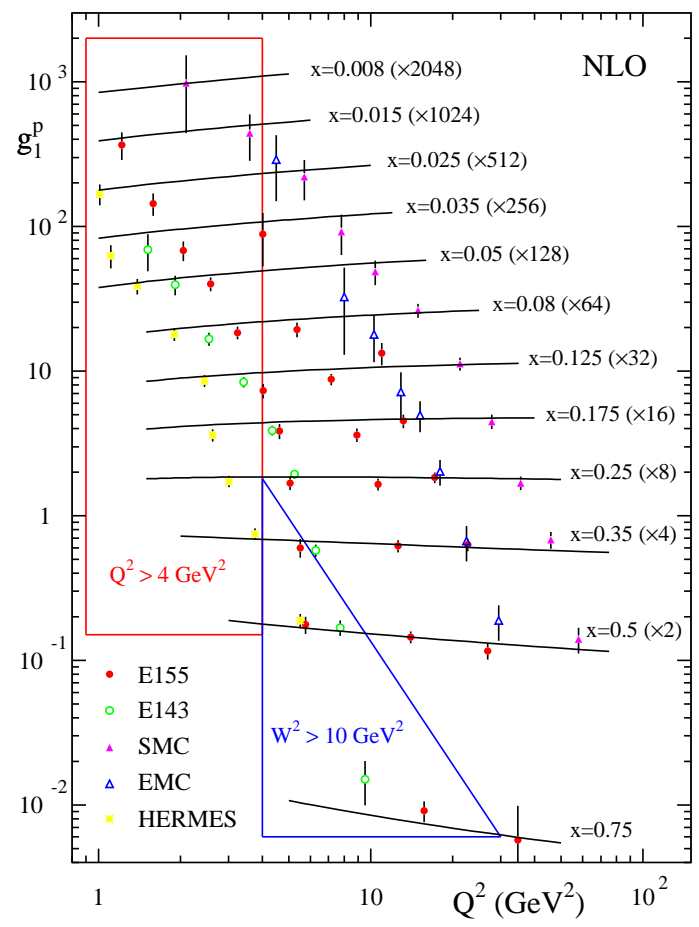

Figure 1. Available information on $g_{1}\left(x, Q^{2}\right)$ as collected by fixed-target experiments ${ }^{1}$ compared to results of a NLO QCD fit (solid lines). The indicated rectangular and triangular regions contain data which would not pass kinematical cuts of $Q^{2}>4 \mathrm{GeV}^{2}$ and $W^{2}>10 \mathrm{GeV}^{2}$, respectively, typically imposed in all fits to unpolarized DIS data.

the leading-twist approximation are supposed to work, can shed light on the possible size of unwanted higher-twist contributions in presently available sets of polarized parton distributions.

\section{Spin Physics at RHIC with Longitudinal Polarization}

\subsection{Prerequisites}

The QCD-improved parton model has been successfully applied to many high energy scattering processes. The predictive power of perturbative QCD follows from the universality of the parton distributions and fragmentation functions. Once extracted from data they can be used to make definite predictions for other processes. This property is based on the factorization theorem where a cross section is written as a convolution of perturbatively calculable partonic hard scattering coefficients $d \hat{\sigma}_{a b}^{c}$ and appropriate parton densities $f_{a, b}$ and/or fragmentation functions $D_{c}^{H}$. To be specific, let us consider the inclusive pro- 
duction of a hadron $H$, e.g., a pion, in unpolarized proton-proton collisions:

$$
\frac{d \sigma^{H}}{d \Gamma}=\sum_{a b c} \int d x_{a} d x_{b} d z f_{a}\left(x_{a}, \mu\right) f_{b}\left(x_{b}, \mu\right) \frac{d \hat{\sigma}_{a b}^{c}}{d \Gamma}\left(x_{a}, x_{b}, z, \Gamma, \mu\right) D_{c}^{H}(z, \mu) .
$$

Here, $\Gamma$ stands for any appropriate set of kinematical variables like the transverse momentum $p_{T}$ and/or rapidity $y$ of the observed hadron. The functions $f_{a, b}$ and $D_{c}^{H}$ embody non-perturbative physics. However, once they are known at some initial scale $\mu_{0}$, their scale $\mu$-dependence is calculable perturbatively via a set of evolution equations. The factorization scale $\mu$, introduced on the r.h.s. of (1), separates long- and short-distance phenomena. $\mu$ is completely arbitrary but usually chosen to be of the order of the scale characterizing the hard interaction, for instance $p_{T}$. Since the l.h.s. of (1) has to be independent of $\mu$ (and other theoretical conventions), any residual dependence of the r.h.s. on the actual choice of $\mu$ gives an indication of how well the theoretical calculation is under control and can be trusted. In particular, leading order (LO) estimates suffer from a strong, uncontrollable scale dependence and hence are not sufficient for comparing theory with data. Figure 2 shows a typical factorization scale dependence for various processes and experiments as a function of $p_{T}$. Clearly, the situation is only acceptable at collider experiments where one can access $p_{T} \gtrsim 5 \mathrm{GeV} \ll \sqrt{S} / 2=p_{T}^{\max }\left(\sqrt{S}\right.$ is the available c.m.s. energy). $p_{T}$ values of about 1-2 GeV, accessible at fixed-target experiments, are not sufficient to provide a large enough hard scale to perform perturbative calculations reliably. For simplicity we have not distinguished between renormalization and initial/final-state factorization scales in (1) which can be chosen differently.

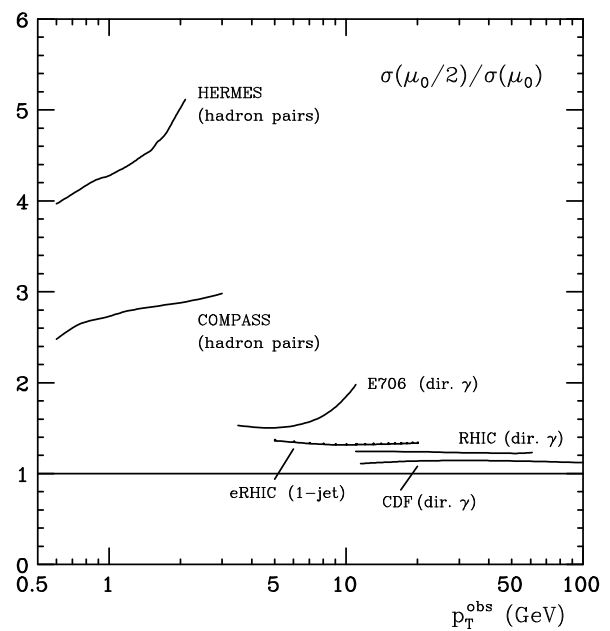

Figure 2. Typical factorization scale dependence for various processes and experiments as a function of $p_{T}$. Shown is the cross section ratio for two choices of scale, $p_{T}$ and $p_{T} / 2$. 
So far we have neglected the spin information contained in parton densities (and fragmentation functions). Eq. (1) can be easily extended to incorporate polarization by replacing all unpolarized quantities by their spin-dependent counterparts, like, for instance, $f_{a, b} \rightarrow \Delta f_{a, b}$ and $d \hat{\sigma}_{a b}^{c} / d \Gamma \rightarrow d \Delta \hat{\sigma}_{a b}^{c} / d \Gamma$. If a hard-scattering process with incoming protons having definite spin orientations is studied, as at RHIC, one gains access to the spin distributions of quarks and gluons in a longitudinally (or transversely) polarized proton. In practice, spin experiments measure not the polarized cross section, $d \Delta \sigma / d \Gamma$, itself, but the spin asymmetry, which is given by the ratio of the polarized and unpolarized cross sections, e.g., for our example, Eq. (1), it reads

$$
A_{\mathrm{LL}}^{H} \equiv \frac{d \Delta \sigma^{H} / d \Gamma}{d \sigma^{H} / d \Gamma}
$$

To denote the type of polarization of the colliding hadrons in (2) we use the subscripts ' $L=$ longitudinal' and ' $T=$ transverse'. At RHIC one can also study doubly transverse spin asymmetries, $A_{\mathrm{TT}}$, and single spin asymmetries $A_{\mathrm{L}}, A_{\mathrm{T}}$ (the latter is often called $A_{\mathrm{N}}$ ) where only one of the protons is polarized.

\subsection{Accessing $\Delta g$}

The main thrust of the RHIC spin program ${ }^{2}$ is to pin down the so far elusive gluon helicity distributions $\Delta g(x, \mu)$. The strength of RHIC is the possibility to probe $\Delta g(x, \mu)$ in a variety of hard processes $^{2}$, in each case at sufficiently large $p_{T}$ where perturbative QCD is expected to work. This not only allows to determine the $x$-shape of $\Delta g(x, \mu)$ for $x \gtrsim 0.01$ but also verifies the universality property of polarized parton densities for the first time. In the following we review the status of theoretical calculations for processes sensitive to $\Delta g$, experimental aspects can be found, e.g., in ${ }^{3}$.

The 'classical' tool for determining the gluon density is high- $p_{T}$ prompt photon production due to the dominance of the LO Compton process, $q g \rightarrow \gamma q$. Exploiting this feature, both RHIC experiments, PHENIX and STAR, intend to use this process for a measurement of $\Delta g$. Apart from 'direct' mechanisms like $q g \rightarrow \gamma q$, the photon can also be produced by a parton, scattered or created in a hard QCD reaction, which fragments into the photon. Such a contribution naturally arises in a QCD calculation from the necessity of factorizing finalstate collinear singularities into a photon fragmentation function $D_{c}^{\gamma}$. However, since photons produced through fragmentation are always accompanied by hadronic debris, an 'isolation cut' imposed on the photon signal in experiment, e.g., a 'cone', strongly reduces such contributions to the cross section.

The NLO QCD corrections to the direct (non-fragmentation) processes have been calculated $\mathrm{in}^{4}$ and lead to a much reduced factorization scale dependence 


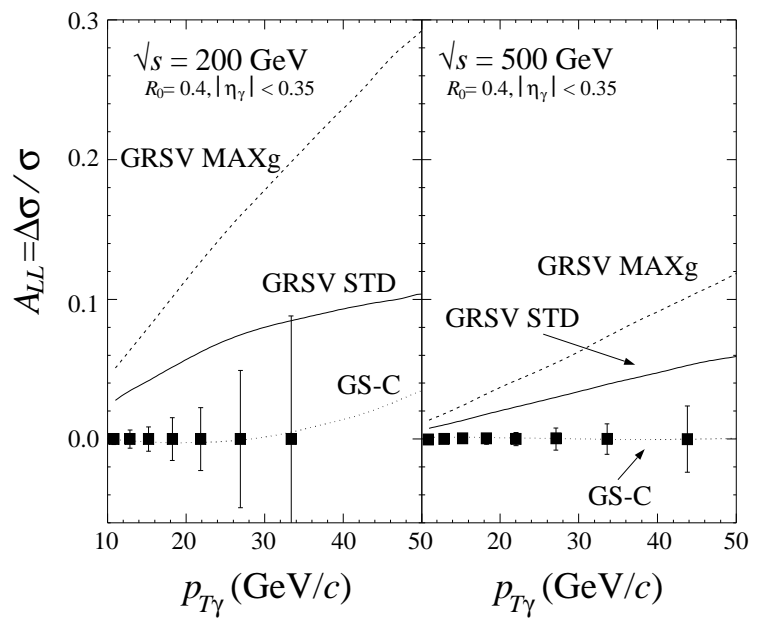

Figure 3. $A_{\mathrm{LL}}$ for prompt photon production in NLO QCD as a function of $p_{T}$ for different sets of parton densities. The 'error bars' indicate the expected statistical accuracy for the PHENIX experiment. Figure taken from ${ }^{6}$.

as compared to LO estimates. In addition, Monte Carlo codes have been developed $^{5,6}$, which allow to include various isolation criteria and to study photon-plus-jet observables. The latter are relevant for $\Delta g$ measurements at $\mathrm{STAR}^{2,3}$. Since present comparisons between experiment and theory are not fully satisfactory in the unpolarized case, in particular in the fixed-target regime, considerable efforts have been made to push calculations beyond the NLO of QCD by including resummations of large logarithms ${ }^{7}$. It is hence not unlikely that a better understanding of prompt photon production can be achieved soon. Figure 3 shows $A_{\mathrm{LL}}^{\gamma}$ as predicted by a NLO QCD calculation ${ }^{6}$ as a function of the photon's transverse momentum. The rapidity cut $|\eta| \leq 0.35$ matches the acceptance of the PHENIX detector. The important result is that the expected statistical errors are considerably smaller than the changes in $A_{\mathrm{LL}}^{\gamma}$ due to different spin-dependent gluon densities over a wide range of $p_{T}$. RHIC should be able to probe $\Delta g$ in prompt photon production.

Jets are another key-process to pin down $\Delta g$ at RHIC: they are copiously produced at $\sqrt{S}=500 \mathrm{GeV}$, even at high $p_{T}, 15 \lesssim p_{T} \lesssim 50 \mathrm{GeV}$, and gluoninduced $g g$ and $q g$ processes are expected to dominate in accessible kinematical regimes. Due to limitations in the angular coverage, jet studies will be performed by STAR only. PHENIX can alternatively look for high- $p_{T}$ leading hadrons, such as pions, whose production proceeds through the same partonic subprocesses as jet production. Hadrons have the advantage that they can be studied also at $\sqrt{S}=200 \mathrm{GeV}$ and down to lower values in $p_{T}$ than jets as they do not require the observation of clearly structured 'clusters' of particles 


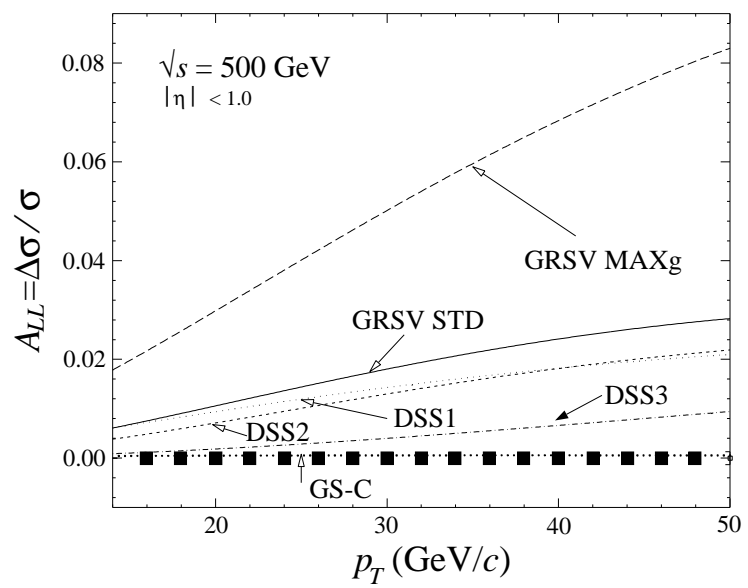

Figure 4. As in Fig. 3 but now for high- $p_{T}$ jet production. The 'error bars' are for the STAR experiment taking into account its acceptance. Figure taken from ${ }^{8}$.

(jets). On the downside, they require a fragmentation function in the theoretical description, cf. Eq. (1), which is, however, fairly well constrained by $e^{+} e^{-}$ data. It should be emphasized that in the unpolarized case, the comparison between NLO theory predictions with jet production data from the Tevatron is extremely successful.

The NLO QCD corrections to polarized jet production are available as a Monte Carlo code ${ }^{8}$. Apart from a significant reduction of the scale dependence, they are also mandatory for realistically matching the procedures used in experiment in order to group final-state particles into jets. For single-inclusive high- $p_{T}$ hadron production the task of computing the NLO corrections has been completed only very recently ${ }^{9}$. Figure 4 shows $A_{\mathrm{LL}}$ for single-inclusive jet production at the NLO level as a function of the jet $p_{T}$. A cut in rapidity, $|\eta| \leq 1$, has been applied in order to match the acceptance of STAR. The asymmetries turn out to be smaller than for prompt photon production, but thanks to the much higher statistics one can again easily distinguish between different spin-dependent gluon densities. Very similar results are obtained for single-inclusive pion production ${ }^{9}$.

The last process which exhibits a strong sensitivity to $\Delta g$ is heavy flavor production. Here, the LO gluon-gluon fusion mechanism, $g g \rightarrow Q \bar{Q}$, dominates unless $p_{T}$ becomes rather large. Unpolarized calculations have revealed that NLO QCD corrections are mandatory for a meaningful quantitative analysis. In the polarized case they have been completed only very recently for singleinclusive heavy quark production ${ }^{10}$. Again, one observes a strongly reduced scale dependence for charm and bottom production at RHIC energies. It turns 


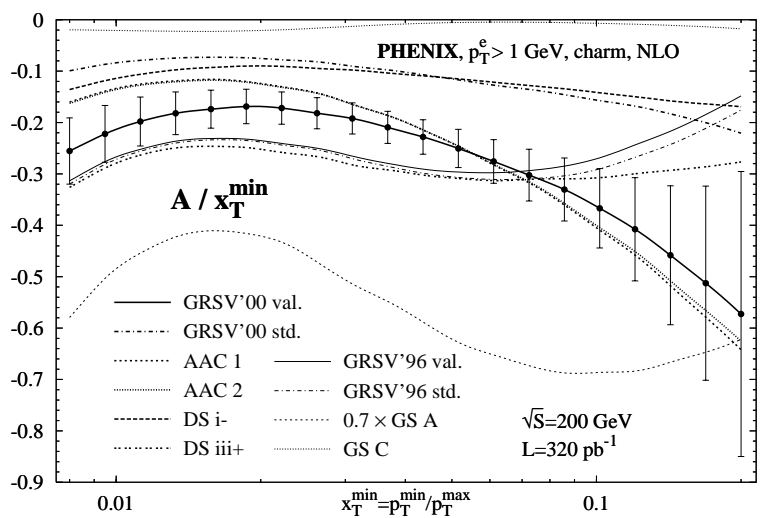

Figure 5. NLO single-inclusive charm production asymmetry (rescaled by $1 / x_{T}^{\mathrm{min}}$ ) as a function of $x_{T}^{\min } \equiv p_{T}^{\min } / p_{T}^{\max }$ for different sets of parton densities. The 'error bars' are for the PHENIX experiment and include a detection efficiency for the channel $c \rightarrow e X$ as modeled by PYTHIA. Figure taken from ${ }^{10}$.

out that the major theoretical uncertainty stems from the unknown precise values for the heavy quark masses ${ }^{10}$. Since the heavy quark mass already sets a large scale, one can perform calculations for small transverse momenta or even for total cross sections which give access to the gluon density at smaller $x$-values than relevant for jet or prompt photon production.

Heavy flavors are not observed directly at RHIC but only through their decay products. Possible signatures for charm/bottom quarks at PHENIX are inclusive-muon or electron tags or $\mu e$-coincidences. The latter provide a much better $c / b$-separation which is an experimental problem. In addition, lepton detection at PHENIX is limited to $|y| \leq 0.35$ and $1.2 \leq|y| \leq 2.4$ for electrons and muons, respectively. Since heavy quark decays to leptons proceed through different channels and have multi-body kinematics, it is a non-trivial task to relate, e.g., experimentally observed $p_{T}$-distributions of decay muons to the calculated $p_{T}$-spectrum of the produced heavy quark. One possibility is to model the decay with the help of standard event generators like PYTHIA ${ }^{11}$ by computing probabilities that a heavy quark with a certain $\left(p_{T}, y\right)$ is actually seen within the PHENIX acceptance for a given decay mode. Figure 5 shows a prediction for the charm production asymmetry $A_{\mathrm{LL}}$ at PHENIX in NLO QCD for the inclusive-electron tag. The sensitivity to $\Delta g$ is less pronounced than for the processes discussed above. It remains to be checked if heavy flavor production at RHIC can be used to extend the measurement of $\Delta g$ towards smaller $x$-values. Also, progress has to be made to solve the longstanding puzzle that the inclusive $b$-rate as predicted by QCD is too small in unpolarized $p \bar{p}, e p$, and $\gamma \gamma$ collisions $^{12}$. 


\subsection{Further Information on $\Delta q$ and $\Delta \bar{q}$}

Inclusive DIS data only provide information on the sum of quarks and antiquarks for each flavor, i.e., $\Delta q+\Delta \bar{q}$. At RHIC a separation of $\Delta u, \Delta \bar{u}, \Delta d$, and $\Delta \bar{d}$ can be achieved by studying $W^{ \pm}$-boson production. Exploiting the parity-violating properties of $W^{ \pm}$-bosons, it is sufficient to measure a single spin asymmetry, $A_{\mathrm{L}}^{W}$, with only one of the colliding protons being longitudinally polarized. The idea is to study $A_{\mathrm{L}}^{W}$ as a function of the rapidity of the $W$, $y_{W}$, relative to the polarized proton ${ }^{13}$. In LO it is then easy to show ${ }^{13,2}$ that for $W^{+}$-production, $u \bar{d} \rightarrow W^{+}$, and large and positive (negative) $y_{W}, A_{\mathrm{L}}^{W}$ is sensitive to $\Delta u / u(\Delta \bar{d} / \bar{d})$. Similarly, $W^{-}$-production probes $\Delta d / d$ and $\Delta \bar{u} / \bar{u}$. The NLO QCD corrections for $A_{\mathrm{L}}$ as well as the factorization scale dependence are small ${ }^{14}$. Experimental complications ${ }^{2}$ arise, however, from the fact that neither PHENIX nor STAR are hermetic, which considerably complicates the reconstruction of $y_{W}$. The anticipated sensitivity of PHENIX on the flavor decomposed quark and antiquark densities is illustrated in Fig. 6.

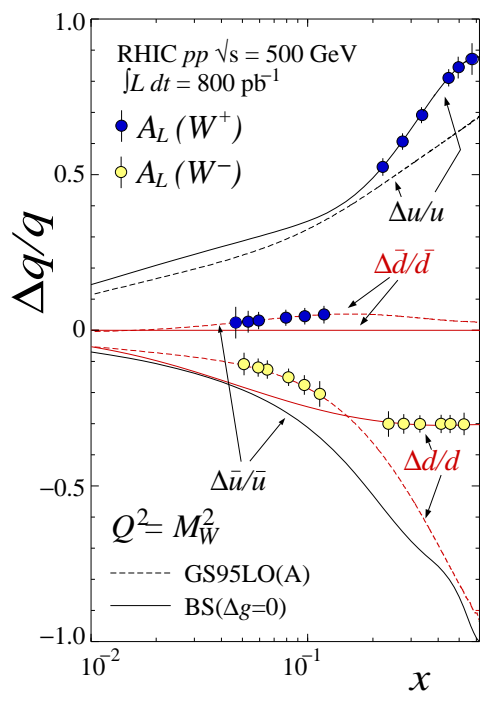

Figure 6. Expected statistical accuracy for $\Delta q / q$ from $A_{\mathrm{L}}$ overlayed on two sets of parton densities. The full [open] circles refer to $A_{\mathrm{L}}\left(W^{+}\right)\left[A_{\mathrm{L}}\left(W^{-}\right)\right]$. Figure taken from ${ }^{2}$.

Semi-inclusive DIS measurements, $e p \rightarrow H X$, are another probe to separate quark and antiquark densities. HERMES has recently published first preliminary results ${ }^{15}$. The accessible $x$-range for the $\Delta q$ and $\Delta \bar{q}$ densities is comparable to that of RHIC, see Fig. 6 , but at scales $Q \simeq 1-2 \mathrm{GeV}$ rather than $M_{W}$. The combination of both measurements can provide an important test of the QCD scale evolution for polarized parton densities. 


\subsection{Towards a Global Analysis of Upcoming Data}

Having available at some point in the near future data on various different reactions, one needs to tackle the question of how to set up a 'global QCD analysis' for spin-dependent parton densities. The strategy is in principle clear from the unpolarized case: an ansatz for the densities at some initial scale $\mu_{0}$, given in terms of some functional form with a set of free parameters, is evolved to a scale $\mu$ relevant for a certain data point. A $\chi^{2}$-value is assigned that represents the quality of the comparison of the theoretical calculation to the experimental point. The parameters are varied until eventually a global minimum in $\chi^{2}$ is reached mutually for all data points. In practice, this approach is not fully viable since the numerical evaluations of the cross sections in NLO QCD are usually time-consuming as they require several tedious integrations. Hence the computing time for a QCD fit easily becomes excessive.

In the unpolarized case, the wealth of DIS data already provides a pretty good knowledge of the parton densities, and reasonable approximations can be made for the most time-consuming processes. For instance, one can absorb all NLO corrections into some pre-calculated 'correction factors' $K$, and simply multiply them in each step of the fit to the LO approximation for the cross sections which can be evaluated much faster. In the polarized case, it is in general not at all clear whether such a strategy will work. Here, parton densities are known with much less accuracy so far. It is therefore not possible to use $K$-factors reliably. In addition, spin-dependent parton densities as well as partonic cross sections may oscillate, i.e., have zeros, in the kinematical regions of interest such that predictions at LO and the NLO can show marked differences. Clearly, in the polarized case the goal must be to find a way of implementing efficiently, and without approximations, the exact NLO expressions for all relevant hadronic cross sections. A very simple and straightforward method based on 'double Mellin transformations' was proposed in ${ }^{16}$.

Recently, its actual practicability and usefulness in a global QCD analysis has been demonstrated ${ }^{17}$ in a case study based on fictitious prompt photon data.

\section{Spin Physics at RHIC with Transverse Polarization}

At RHIC one can also study collisions of transversely polarized protons ${ }^{2}$ giving access to the completely unmeasured leading-twist 'transversity' densities ${ }^{18} \delta f$. Upon expressing transversely polarized eigenstates as superpositions of helicity eigenstates, $\delta f$ reveals its helicity-flip, chiral-odd nature which explains its elusiveness. Other striking features of $\delta f$ are that no transversity gluon density is possible for spin- $1 / 2$ targets and the fact that $\delta f(x, \mu)$ 'evolves away' at all $x$ with increasing scale $\mu$. 


\subsection{Double Spin Asymmetries $\mathbf{A}_{\mathrm{TT}}$}

The requirement of helicity conservation in hard scattering processes implies that chirality has to be flipped twice in order to be sensitive to transversity. One possibility is to have both colliding protons transversely polarized and to study double spin asymmetries $A_{\mathrm{TT}}$. Since gluons play an important or even dominant role in almost all unpolarized production processes, $A_{\mathrm{TT}}$ is expected to be very small in general ${ }^{18}$. In addition, $A_{\mathrm{TT}}$ is further diminished by the requirement of a double chirality flip, which excludes some of the 'standard' $2 \rightarrow 2$ amplitudes to contribute, whereas the remaining ones are color-suppressed. In principle, the most favorable reaction for determining transversity is the Drell-Yan process, $p p \rightarrow \mu^{+} \mu^{-}$, which has no gluonic contribution in LO. However, a recent NLO study of upper bounds for $A_{\mathrm{TT}}$, due to Soffer's inequality ${ }^{19}, 2|\delta f| \leq f+\Delta f$, has revealed that the limited muon acceptance for PHENIX threatens to make a measurement of transversity elusive in this channel ${ }^{20}$. Recently is has been shown that, although $A_{\text {TT }}$ is rather minuscule, jet and prompt photon production can be a useful tool to decipher transversity at RHIC ${ }^{21}$, see Fig. 7. It should be noted that NLO QCD corrections for these processes are still lacking. Needless to say that such measurements are challenging, albeit not completely impossible. The experimental finding of a much larger $A_{\mathrm{TT}}$ than theoretically expected would constitute a new spin puzzle.
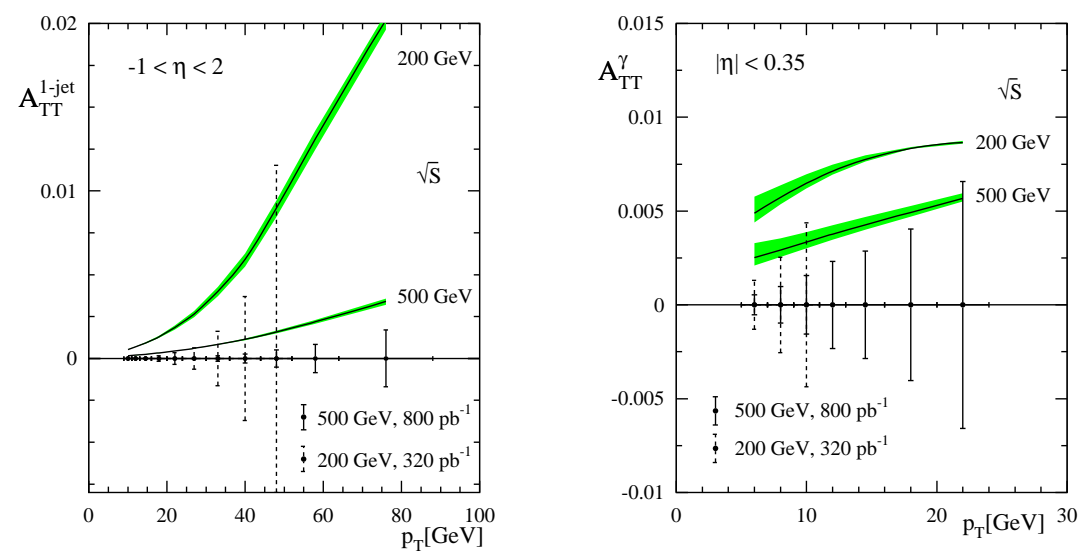

Figure 7. Left: upper bound for $A_{\mathrm{TT}}$ for single-inclusive jet production at RHIC as a function of $p_{T}$. Jet rapidities are integrated over the detector acceptance $(-1 \leq \eta \leq 2)$. The shaded bands represent the uncertainties due to variations of the factorization scale in the range $p_{T} / 2 \leq \mu \leq 2 p_{T}$. The expected statistical accuracy is indicated as 'error bars'. Right: same as on the l.h.s. but now for prompt photon production. The photon rapidity has been integrated over the range $|\eta| \leq 0.35$. Figure taken from ${ }^{21}$. 
Alternative observables have only one transversely polarized initial hadron and a fragmentation process in the final state that is sensitive to transverse polarization ${ }^{18}$. Several processes have been identified as being potentially suitable for a measurement of $\delta f$ : the production of transversely polarized $\Lambda$ hyperons ${ }^{22}$, the asymmetry in the $p_{T}$-distribution of a hadron in a jet around the jet axis $^{23}$ ('Collins effect'), or the interference between $s$ - and $p$-waves of a two pion system ${ }^{24}$ ('interference fragmentation'). Such measurements can be also carried out at HERMES and COMPASS. However, in all cases involving these novel fragmentation effects, the analyzing power is a priori unknown and may well be small, and often there are competing mechanisms for generating the same observable that do not involve transversity.

\subsection{Single Spin Asymmetries $\mathrm{A}_{\mathrm{N}}$}
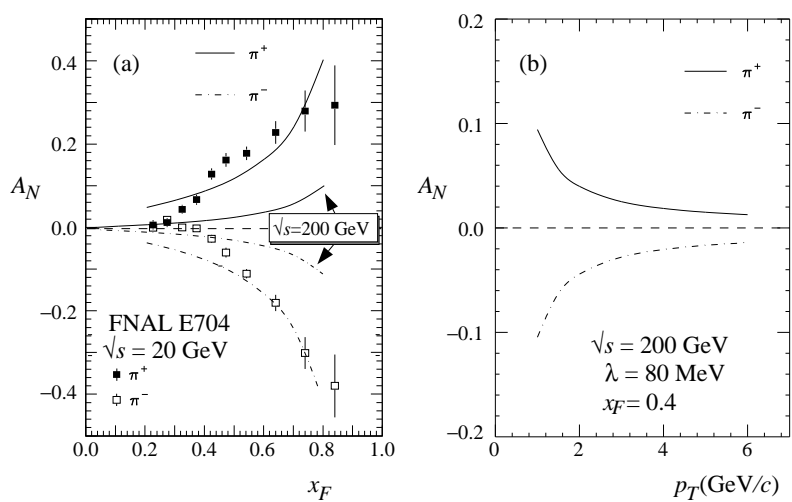

Figure 8. (a): $A_{\mathrm{N}}$ for pion production in $p p$ collisions at $\sqrt{S}=20 \mathrm{GeV}$ compared to E704 data. Predictions for $\mathrm{RHIC}^{25}, \sqrt{S}=200 \mathrm{GeV}$, for $p_{T}=4 \mathrm{GeV}$ are superimposed. (b): $p_{T}$-dependence of $A_{\mathrm{N}}$ for RHIC at $x_{F}=0.4$. Figure taken from ${ }^{2}$.

Surprisingly large single transverse spin asymmetries $A_{\mathrm{N}}$ have been observed over many years in low-energy fixed-target experiments ${ }^{18}$, e.g, for $p p \rightarrow \pi X$. At the leading-twist level for standard ( $k_{T}$-integrated) parton distributions $A_{N}$ is exactly zero. One possible explanation is based on a generalized factorization theorem in perturbative QCD, where a non-vanishing $A_{\mathrm{N}}$ can arise, for instance, as a convolution of some calculable hard-scattering function with an ordinary twist-two parton density from the unpolarized proton and a new twist-three quark-gluon correlation function characterizing the polarized hadron ${ }^{25}$. In a simple model for these correlation functions, which are believed to give the dominant contribution to $A_{\mathrm{N}}$ in this approach, a qualitative description of the available data is possible ${ }^{25}$, see Fig. 8 , and various predictions have been made which can be tested at RHIC. In particular, at 
RHIC one should see the fall-off of $A_{\mathrm{N}}$ with $p_{T}$ associated with its higher-twist nature, see Fig. 8.

An alternative approach to $A_{\mathrm{N}}$ introduces intrinsic transverse momentum $k_{T}$ into distribution and fragmentation functions ${ }^{18}$. This opens a Pandora's box with many new and unknown functions. Each of three possible mechanisms on its own can account for $A_{\mathrm{N}}$ data $^{18}$. Needless to say that it is very difficult to disentangle all these effects. RHIC, with the help of other experiments, can help to shed some light on the origin of transverse single spin asymmetries.

\section{Exploring Physics Beyond the Standard Model}

Spin observables are also an interesting tool to uncover important new physics. One idea is to study single spin asymmetries $A_{L}$ for large- $p_{T}$ jets. In the standard model $A_{L}$ can be only non-zero for parity-violating interactions, i.e., QCD-electroweak interference contributions, which are fairly small. The existence of new parity-violating interactions could lead to sizable modifications ${ }^{26}$ of $A_{L}$. Possible candidates are new quark-quark contact interactions, characterized by a compositeness scale $\Lambda$. RHIC is surprisingly sensitive to quark substructure at the $2 \mathrm{TeV}$ scale, and is competitive with the Tevatron despite the much lower c.m.s. energy ${ }^{26}$. Other candidates for new physics are possible new gauge bosons, e.g., a leptophobic $Z^{\prime}$. Of course, high luminosity and precision as well as a good knowledge of polarized and unpolarized parton densities and of the standard model 'background' are mandatory. For details, see ${ }^{2,26}$.

\section{Summary and Outlook}

With first data from RHIC hopefully starting to roll in soon, we can address many open, long-standing questions in spin physics like the longitudinally polarized gluon density or transversity. With data from many different processes taken at high energies where perturbative QCD should be at work, a first global analysis of spin-dependent parton densities will be possible. At the end of RHIC we certainly have a much improved knowledge of the spin structure of the nucleon, and, perhaps, the next 'spin surprise' is just round the corner. Future projects like the $\mathrm{EIC}^{27}$, which is currently under scrutiny, would help to further deepen our understanding by probing aspects of spin physics not accessible in hadron-hadron collisions. The structure function $g_{1}$ at small $x$ or the spin content of circularly polarized photons are just two examples.

\section{Acknowledgments}

I am grateful to the organizers for inviting me to an interesting and lively workshop in Charlottesville. 


\section{References}

1. For a review on experimental efforts and results in polarized deep-inelastic scattering, see: E. Hughes and R. Voss, Annu. Rev. Nucl. Part. Sci. 49, 303 (1999).

2. For a review on RHIC spin, see: G. Bunce, N. Saito, J. Soffer, and W. Vogelsang, Annu. Rev. Nucl. Part. Sci. 50, 525 (2000).

3. M. Grosse Perdekamp, these proceedings.

4. L.E. Gordon and W. Vogelsang, Phys. Rev. D48, 3136 (1993); A.P. Contogouris et al., Phys. Lett. B304, 329 (1993); Phys. Rev. D48, 4092 (1993).

5. L.E. Gordon, Nucl. Phys. B501, 197 (1997); L.E. Gordon and G.P. Ramsey, Phys. Rev. D59, 074018 (1999); S. Chang et al., Phys. Rev. D58, 074002 (1998).

6. S. Frixione and W. Vogelsang, Nucl. Phys. B568, 60 (2000).

7. E. Laenen et al., Phys. Lett. B438, 173 (1998); Phys. Rev. Lett. 84, 4296 (2000); S. Catani et al., JHEP 9807, 024 (1998); 9903, 025 (1999).

8. D. de Florian et al., Nucl. Phys. B539, 455 (1999).

9. D. de Florian, hep-ph/0210442; B. Jäger, M. Stratmann, A. Schäfer, and W. Vogelsang, hep-ph/021100\%.

10. I. Bojak and M. Stratmann, hep-ph/0112276 and work in progress.

11. T. Sjöstrand et al., hep-ph/0108264.

12. Recent results on $b$-production can be found in: F. Sefkow, hep-ex/0109038.

13. P. Chiappetta and J. Soffer, Phys. Lett. B152, 126 (1985); C. Bourrely and J. Soffer, Phys. Lett. B314, 132 (1993); Nucl. Phys. B423, 329 (1994); B445, 341 (1995).

14. A. Weber, Nucl. Phys. B403, 545 (1993); B. Kamal, Phys. Rev. D57, 6663 (1998); T. Gehrmann, Nucl. Phys. B534, 21 (1998).

15. M. Beckmann, these proceedings [hep-ex/0210049].

16. D.A. Kosower, Nucl. Phys. B520, 263 (1998).

17. M. Stratmann and W. Vogelsang, Phys. Rev. D64, 114007 (2001).

18. For a review on transversity, see: V. Barone, A. Drago, and P.G. Ratcliffe, Phys. Rept. 359,1 (2002).

19. J. Soffer, Phys. Rev. Lett. 74, 1292 (1995); D. Sivers, Phys. Rev. D51, 4880 (1995).

20. O. Martin et al., Phys. Rev. D57, 3084 (1998); D60, 117502 (1999).

21. J. Soffer, M. Stratmann, and W. Vogelsang, Phys.Rev. D65, 114024 (2002).

22. D. de Florian et al., Phys.Lett. B439, 176 (1998).

23. J.C. Collins, Nucl. Phys. B396, 161 (1993).

24. J.C. Collins et al., Nucl. Phys. B420, 565 (1994); X. Ji, Phys. Rev. D49, 114 (1994); R.L. Jaffe et al., Phys. Rev. Lett. 80, 1166 (1998); Phys. Rev. D57, 5920 (1998).

25. J. Qiu and G. Sterman, Phys. Rev. Lett. 67, 2264 (1991); Nucl. Phys. B378, 51 (1992); Phys. Rev. D59, 014004 (1999).

26. P. Taxil and J.-M. Virey, Phys. Lett. B364, 181 (1995); B383, 355 (1996); B441, 376 (1998); Phys. Rev. D55, 4480 (1997).

27. For information concerning the EIC project including the EIC Whitepaper, see: http://www.bnl.gov/eic. 